\title{
Pediatric Retinal Detachment in Indonesia: Clinical Characteristics, Risk Factors, and Treatment Outcomes
}

\author{
Irawati Irfani, Arief S. Kartasasmita \\ Universitas Padjadjaran/Cicendo National Eye Hospital, Bandung, Indonesia \\ Email: irawati.irfani@unpad.ac.id
}

How to cite this paper: Irfani, I. and Kartasasmita, A.S. (2017) Pediatric Retinal Detachment in Indonesia: Clinical Characteristics, Risk Factors, and Treatment Outcomes. Open Journal of Ophthalmology, 7 , 249-255.

https://doi.org/10.4236/ojoph.2017.74033

Received: August 19, 2017

Accepted: September 26, 2017

Published: September 29, 2017

Copyright ( 92017 by authors and Scientific Research Publishing Inc. This work is licensed under the Creative Commons Attribution International License (CC BY 4.0).

http://creativecommons.org/licenses/by/4.0/

\section{cc) (i) Open Access}

\begin{abstract}
Purpose: To describe the clinical features and risk factors of pediatric retinal detachment among patients in Indonesia. Methods: This is a retrospective study involving 46 eyes of 34 children (younger than 18 years) diagnosed with pediatric retinal detachment. A detailed history was taken and a complete ophthalmic examination and a systemic examination were performed as required. Clinical characteristics, risk factors, and treatment choices were noted. Retinal detachment was categorized as tractional, exudative, or rhegmatogenous. Results: Mean patient age was 8.5 years (range, $0-18$ years). Most patients (70\%) were boys. Twelve (35\%) patients had bilateral involvement at presentation. Tractional retinal detachment was found in 17 eyes (37\%) and in this study was caused by retinopathy of prematurity (grade IV-V) in all cases. Exudative retinal detachment was found in 12 eyes (26\%), the most common causes of which were panuveitis and Coat's disease (both 50\%). Rhegmatogenous retinal detachment was found in 17 eyes (37\%), the most common risk factor for which was trauma (58\%). Conclusions: Different approaches are needed to treat pediatric retina detachment in patients with different risk factors. Recognition of risk factors and early management will help to prevent childhood blindness due to retinal detachment.
\end{abstract}

\section{Keywords}

Pediatric Retinal Detachment, Clinical Characteristics, Risk Factors

\section{Introduction}

Pediatric retinal detachment (RD) is a devastating ophthalmic condition if not properly treated. The prevalence of $\mathrm{RD}$ is reported as 12.4 cases per 100,000 in- 
dividuals, of which $3.2 \%-5.6 \%$ (0.38 - 0.69 cases per 100,000 individuals) are of pediatric age [1] [2] [3] [4]. Management of pediatric RD remains challenging due to the viscosity of pediatric vitreous, the difficulty of scleral buckling in developing eyes, and the risk of proliferative retinopathy caused by a raised immune response [2] [3] [5].

The surgical management of pediatric $\mathrm{RD}$ is difficult; therefore, prevention of the condition is important. An improved understanding of the etiological risk factors associated with the disease may help to avoid visual morbidity and childhood blindness. In addition, early detection and diagnosis is essential because timing is a crucial factor in the success of RD management. This is especially important in developing countries, where individuals often seek treatment too late for viable surgical management.

We performed this study to describe the clinical characteristics and most common risk factors for pediatric RD in the Cicendo National Eye Hospital $(\mathrm{CNEH})$, as the top referral eye hospital in Indonesia.

\section{Methods}

This is a retrospective descriptive study utilizing hospital medical records of patients diagnosed with RD in 2013. All new patients under 18 years of age and diagnosed with $\mathrm{RD}$ by the consultant in the vitreoretinal unit were included. Age, sex, laterality, visual acuity, and type of $\mathrm{RD}$ were recorded. $\mathrm{RD}$ sub-types were classified by etiology, age group, risk factors, and management

\section{Result}

Retinal detachment was observed in 46 eyes of 34 pediatric patients. The clinical characteristics are shown in Table 1.

\subsection{Characteristics of Retinal Detachment}

The retinal detachment characteristics are shown in Table 2. The mean age of patients in this study was 8.5 years (range: 0 - 18 years), with $70 \%$ of cases being male. Twelve (35\%) patients had bilateral involvement, with tractional RD and exudative RD affecting eight and four patients, respectively. Visual acuity determination was difficult because of age, but most cases had light perception (41\%) followed by hand movement detection (29\%). Visual acuity measurement was not possible for 13 (38\%) patients. When stratified by RD sub-type, most patients had unilateral rhegmatogenous $\mathrm{RD}$ (37\%) and bilateral tractional $\mathrm{RD}$ (37\%).

\subsection{Etiology, Risk Factors, and Management of Pediatric Retinal Detachment}

The overall frequency of RD was highest in the 0 - 5 year age group, with tractional $\mathrm{RD}$ the most common sub-type (89\%) in these patients. Retinopathy of prematurity (ROP) stage IV - V was the sole etiological factor in all cases of 
Table 1. Clinical characteristic of retinal detachment.

\begin{tabular}{cc}
\hline Characteristics & Number/(\%) $(\mathrm{n}=46$ eyes/34 patients) \\
\hline $\begin{array}{c}\text { Age (mean [years] }) \\
(\text { range [years] })\end{array}$ & 0.5 \\
Sex $(\mathrm{n}=34)$ & \\
Male & $24(70 \%)$ \\
Female & $10(29 \%)$ \\
Lateralization $(\mathrm{n}=34)$ & \\
Unilateral & $22(65 \%)$ \\
Bilateral & $12(35 \%)$ \\
RD sub-type $(\mathrm{n}=46)$ & \\
Tractional & $17(37 \%)$ \\
Exudative & $12(26 \%)$ \\
Rhegmatogenous & $17(37 \%)$ \\
\end{tabular}

Table 2. Clinical characteristics, etiology, and other risk factors stratified by retinal detachment sub-type.

\begin{tabular}{cccc}
\hline & $\begin{array}{c}\text { Tractional } \\
(\mathrm{n}=17 \text { eyes/9 patients) }\end{array}$ & $\begin{array}{c}\text { Exudative } \\
(\mathrm{n}=12 \text { eyes/8 patients) }\end{array}$ & $\begin{array}{c}\text { Rhegmatogenous } \\
(\mathrm{n}=17 \text { eyes/17 patients })\end{array}$ \\
\hline Age (years) & & & \\
$0-5$ & $16 / 8(89 \%)$ & $4 / 2(33 ; 25 \%)$ & $3(17 \%)$ \\
$6-10$ & - & $4 / 2(33 ; 25 \%)$ & $7(41 \%)$ \\
$11-15$ & $1 / 1(11 \%)$ & $4 / 4(33 ; 50 \%)$ & $3(17 \%)$ \\
$16-18$ & - & - & $4(23 \%)$ \\
Sex & & & $12(70 \%)$ \\
Male & $6(67 \%)$ & $6(75 \%)$ & $5(30 \%)$ \\
Female & $3(33 \%)$ & $2(25 \%)$ & \\
Etiology \& risk factors & & & \\
ROP stage IV - V & $17 / 9(100 \%)$ & & $10(58 \%)$ \\
Coat's disease & & $4 / 4(33 ; 50 \%)$ & $4(23 \%)$ \\
Panuveitis & & & $1(7 \%)$ \\
Trauma & & & \\
Myopia & & & \\
Intraocular surgery & & & \\
Unknown & & & \\
\hline
\end{tabular}

tractional RD.

By contrast, exudative $\mathrm{RD}$ was most commonly observed in the 11 - 15 year age group, with panuveitis and Coat's disease prevalent causes. Panuveitis is associated with infectious disease including tuberculosis (TB) and TORCH (Toxoplasma, Rubella, Cytomegalovirus and Herpes), as infection remains a leading cause of ocular inflammation in Indonesia. Coat's disease may occur in the first decade of life and has devastating visual sequelae if not managed early in the 
Table 3. Management of pediatric retinal detachment.

\begin{tabular}{|c|c|c|c|}
\hline Management & Tractional RD & Exudative RD & Rhegmatogenous RD \\
\hline Observation & $9(100 \%)$ & $4(50 \%)$ & $11(64 \%)$ \\
\hline Medication & & $4(50 \%)$ & \\
\hline \multicolumn{4}{|l|}{ Surgical } \\
\hline Laser photocoagulation & & & $1(6 \%)$ \\
\hline Scleral buckle (SB) & & & $1(6 \%)$ \\
\hline Vitrectomy (VPP) & & & $3(18 \%)$ \\
\hline $\mathrm{VPP}+\mathrm{SB}$ & & & $1(6 \%)$ \\
\hline \multicolumn{4}{|l|}{ Anatomical result } \\
\hline Attached retina & & & $1(16 \%)$ \\
\hline Re-detached & & & $5(84 \%)$ \\
\hline
\end{tabular}

disease process. In this study, we noted four patients with Coat's disease (11 - 15 years) that could not be treated with photocoagulation or vitreoretinal surgery because of advanced disease progression.

Rhegmatogenous $\mathrm{RD}$ was found in $37 \%$ of eyes, with $41 \%$ of these occurring in the 6 - 10 year age group. Trauma (58\%) was the most common risk factor for this RD sub-type.

In this study, the primary management modality for $\mathrm{RD}$ was observation because most cases presented too late for effective medical or surgical treatment. Medication was given for cases of exudative $\mathrm{RD}$ with panuveitis as a risk factor. Vitreoretinal surgery was performed in $36 \%$ of cases; however, most had poor anatomical outcomes. Table 3 shows the detail management of retinal detachment recorded.

\section{Discussion}

This study characterizes pediatric RD in a government tertiary referral hospital in Indonesia and highlights some differences in disease patterns compared with developed countries.

We found that ROP was the only cause of tractional RD in this study. Report from study involved of 21 health facilities in Indonesia, had a result of 5.05\% (32/613) of premature babies that were diagnosed with ROP [6]. Similar studies from Mongolia, Malaysia, and Latin America detected more case of ROP [7]. In a report from Malaysia, 20 of 294 premature babies examined, about 7\%, had ROP [8]. According to a 2005 report, two-thirds of the 50,000 children worldwide estimated to be blind from ROP are from Latin America. Unfortunately, more mature infants are developing severe ROP in countries with lower or modest levels of development than those in highly developed countries [7]. Further investigation of this risk factor needs to be prioritized because ROP plays an important factor in pediatric $\mathrm{RD}$ and childhood blindness.

Indonesia has a National Guideline for screening and treatment of ROP. 
Based on data from the General Hospital in Jakarta, ROP prevalence had decreased between 2004 and 2010. In 2007, the reported prevalence of ROP was $21.7 \%$ with $71 \%$ of cases being at Stage 3. Subsequent estimates of prevalence were $14 \%$ and $18 \%$ in 2008 and 2009, respectively [6]. An important extension of the national guidelines means increasing the need for ROP centers in the country. Currently, ROP is only detected and managed at a small number of centers, usually tertiary care hospitals and certain private eye clinics, and many infants at risk do not have access to such facilities. Data from the 2010 ROP workshop reflect the high mortality of premature babies in Indonesia, especially in clinics with minimum neonatal facilities [6]. This study shows that ROP was the sole cause of pediatric tractional $\mathrm{RD}$ at a tertiary referral eye hospital in Indonesia $(\mathrm{CNEH})$. Delay in the detection and treatment of $\mathrm{RD}$ was influenced by inadequate ophthalmic screening of premature babies, combined with parental reluctance to subject their child to potential surgery.

The most common risk factor for exudative $\mathrm{RD}$ was panuveitis secondary to $\mathrm{TB}$ and TORCH infection. The prevalence of TB in Indonesia is high and the estimated prevalence of disease in the pediatric population for 2015 was about 75,000 . [9] In this study, $50 \%$ of cases with exudative RD had panuveitis as an underlying condition and all cases had a positive history of tuberculosis.

We found that another common cause of exudative $\mathrm{RD}$ was Coat's disease. This has been observed in previous case series, with Rumelt noting that $63 \%$ of cases of exudative $\mathrm{RD}$ were caused by the disease [1]. In developing countries such as Indonesia, regular retinal screening in children is a difficult public health strategy to implement. While a white pupil in a child is a diagnostic sign for Coat's disease, usually by this stage the retina has detached and laser photocoagulation is no longer a viable management option. Mjeren followed 15 cases of Coat's disease in the early stages of presentation for up to 28 months. These patients were treated with a combination of laser photocoagulation, cryotherapy, and vitreoretinal surgery. Stable visual outcomes and anatomic improvement were achieved in 12 cases with no enucleation necessary [10]. Comparatively, $50 \%$ of cases of exudative pediatric RD were due to Coat's disease in our cohort, but all presented at a late stage of disease (11 - 15 years of age group). This highlights the importance of regular retinal screening in childhood, as with early recognition and appropriate treatment, anatomical and visual rehabilitation in Coat's disease-associated RD can be achieved [10].

Thirty seven percent cases in this study were of the rhegmatogenous $\mathrm{RD}$ sub-type. Trauma remained the most common cause of RD in the $6-10$ years of age group (58\%), followed by myopia in $23 \%$ of cases. These findings are comparable to those of a study from Saudi Arabia, in which $32 \%$ and $17 \%$ of 152 eyes had rhegmatogenous RD secondary to trauma and myopia, respectively [2]. Similarly, Rumlet noted $41 \%$ and $11 \%$ of cases of $\mathrm{RD}$, secondary to trauma and myopia, respectively [1]. However, in a Taiwanese report, Chang found that $37.5 \%$ of 146 patients below 18 years of age had rhegmatogenous $\mathrm{RD}$ as a result 
of high myopia. In children, traumatic $\mathrm{RD}$ often occurs relatively late due to the support of a well-formed vitreous [11]. In a study of salient risk factors, Wenick found that congenital ocular abnormalities, prior ocular surgery, and trauma are principal causes of pediatric RD. By contrast, for the East Asian and older pediatric populations, myopia alone appears to be a major factor [1] [2] [12].

The surgical management of $\mathrm{RD}$ in our series was challenging because individuals typically presented late, resulting in poor anatomical outcomes. Consequently, retinal re-detachment occurred in $84 \%$ of cases. This is similar to that of an Indian report, in which most cases of pediatric $\mathrm{RD}$ presented late, with macular detachment a common finding. Even though complete retinal re-attachment was achieved, visual recovery was modest for most patients [5] [12].

Different disease risk factors often require different approaches in how the disease is managed. The management of pediatric $\mathrm{RD}$ in developing countries will differ from that of developed nations. It is common in Indonesia for individuals with pediatric RD to present for examination late. Facilities for modern vitreoretinal surgery are typically limited to only a few tertiary healthcare centers in developing countries. Therefore, improving screening programs and parental awareness of the need for swift treatment are both important factors in alleviating this burden of childhood disease.

\section{Conflict of Interest}

The authors declare that they have no conflict of interest.

\section{References}

[1] Rumelt, S., Sarrazin, L., Averbukh, E., Halpert, M. and Hemo, I. (2007) Paediatric vs. Adult Retinal Detachment. Eye, 21, 1473-1478. https://doi.org/10.1038/sj.eye.6702511

[2] Wenick, A.S. and Baranano, D.E. (2012) Evaluation and Management of Pediatric Rhegmatogenous Retinal Detachment. Saudi Journal of Ophthalmology, 26, 255-263.

[3] Gonzales, C.R., Singh, S., Yu, F., Kreiger, A.E., Gupta, A. and Schwartz, S.D. (2008) Pediatric Rhegmatogenous Retinal Detachment: Clinical Features and Surgical Outcomes. Retina, 28, 847-852. https://doi.org/10.1097/IAE.0b013e3181679f79

[4] AL-Zaaidi, S., AL-Rashaed, S., Al-Harthi, E., Al-Kahtani, E. and El-Asrar, A.M.A. (2013) Rhegmatogenous Retinal Detachment in Children 16 Years of Age or Younger. Clinical Ophthalmology, 7, 1001-1014. https://doi.org/10.2147/OPTH.S40056

[5] Wadhwa, N., Venkatesh, P., Sampangi, R. and Garg, S. (2008) Rhegmatogenous Retinal Detachment in Children in India; Clinical Characteristics, Risk Factors, and Surgical Outcomes. Journal of AAPOS, 12, 551-554. https://doi.org/10.1016/j.jaapos.2008.05.002

[6] Sitorus, R., Ari Djatikusumo, G.A., Julie Dewi, B. and Dian Estu, Y. (2011) National Guidelines: Screening and Treatment Retinopathy of Prematurity in Indonesia. In: Association, I.O., Ed., Jakarta.

[7] Tasman, W. (2011) Retinopathy of Prematurity; Do We Still Have a Problem? Arc- 
hives of Ophthalmology, 129, 1083-1086.

https://doi.org/10.1001/archophthalmol.2011.192

[8] Gilbert, C.F.A., Gordillo, L., et al. (2005) Characteristic of Infants with Severe Retinopathy of Prematurity in Countries with Low, Moderate, High Levels of Development: Implication for Screening Programs. Pediatrics, 115, e518-e525.

https://doi.org/10.1542/peds.2004-1180

[9] Global Tuberculosis Report 2016, World Health Organization 2016. http://apps.who.int/iris/bitstream/10665/250441/1/9789241565394-eng.pdf?ua=1

[10] Mrejen, S., Metge, F., Denion, E., Dureau, P., Edelson, C. and Caputo, G. (2008) Management of Retinal Detachment in Coats Disease. Study of 15 Cases. Retina, 28, 26-32. https://doi.org/10.1097/IAE.0b013e31816b3158

[11] Chang, P.-Y., Yang, C.-M., Yang, C.-H., et al. (2005) Clinical Characteristics and Surgical Outcomes of Pediatric Rhegmatogenous Retinal Detachment in Taiwan. American Journal of Ophthalmology, 139, 1067-1072.

https://doi.org/10.1016/j.ajo.2005.01.027

[12] Sarrazin, L., Averbukh, E., Halpert, M., Hemo, I. and Rumelt, S. (2004) Traumatic Pediatric Retinal Detachment; a Comparison between Open and Closed Globe Injuries. American Journal of Ophthalmology, 137, 1042-1049.

https://doi.org/10.1016/j.ajo.2004.01.011 